\title{
A NON-DENSE PLANE CONTINUUM $\dagger$
}

\author{
BY J. H. ROBERTS
}

The author has shown $\ddagger$ that if $M$ denotes a square plus its interior in a plane $S$, then there exists an upper semi-continuous collection $\S G$ of mutually exclusive non-degenerate subcontinua of $M$ filling up $M$ and such that $G$ is homeomorphic with $M$. The present paper gives a continuum $M$ which contains no domain yet which has the above property.

Let $I$ denote the interior of a square $J$ in a plane $S$. Let $G$ be an upper semi-continuous collection of mutually exclusive non-degenerate continua filling $J+I$ such that $G$ is homeomorphic with $J+I$. Since no element of $G$ separates $S$ it follows\| that if $S^{\prime}$ denotes the collection consisting of the elements of $G$ and the points of $S$ not belonging to any element of $G$, then $S^{\prime}$ corresponds to $S$ under a continuous one to one correspondence $U$, and $G$ corresponds to $J+I$. Let $G^{*}$ denote the subcollection containing every element of $G$ which contains a point of $J$. The set $G^{*}$ is a simple closed curve. Moreover every element of $G^{*}$ has in common with $J$ either an arc or a single point. Then there exists $\int$ a continuous one to one correspondence $U_{1}$ between $G^{*}$

$\dagger$ Presented to the Society, August 30, 1929.

$\ddagger$ On a problem of $C$. Kuratowski concerning upper semi-continuous colleclions, Fundamenta Mathematicae, vol. 14 (1929), pp. 96-102.

$\S$ For a definition of this term, and of the notion limit element, see R. L. Moore, Concerning upper semi-continuous collections of continua, Transactions of this Society, vol. 27 (1925), pp. 416-428.

|| See R. L. Moore, loc. cit., Theorem 22.

I This may be shown as follows. Let $s_{1}, s_{2}, s_{3}, \cdots$ denote the maximal arcs which are subsets of $J$ and which belong to some element of $G^{*}$. For each $i$ and $j$ $(i \neq j)$ the set $s_{i} \cdot s_{j}$ is vacuous. Let $v\left(s_{i}\right)$ denote the length of the interval $s_{i}$, and $v(J)$ the length of $J$. Suppose first that $v(J)-\sum_{i=1}^{\infty} v\left(s_{i}\right)$ is a positive number $e$ and let $d$ be a positive number less than $e$. A sequence of segments $t_{1}, t_{2}, t_{3}, \ldots$ can be defined inductively so that (1) for each $i$ there is a $j$ such that $t_{j}$ contains $s_{i}$, (2) no two of the segments $t_{1}, t_{2}, t_{3}, \cdots$ have any point in common, and (3) $\sum_{i=1}^{\infty} v\left(t_{i}\right)$ is less than $v(J)-d$. Since the point set $J-\sum_{i=1}^{\infty} t_{i}$ has positive measure it is uncountable. Now the curve $J$ can be transformed into itself in such a way that the sum of the lengths of the images of the intervals $s_{1}, s_{2}$, $s_{3}, \cdots$ is less than the length of $J$. Hence in any case there is a set of segments 
and $J$ such that for uncountably many points $x$ of $J$ it is true that $U_{1}(x)$ (the element of $G^{*}$ corresponding to the point $x$ ) contains $x$. The correspondence $U_{1}$ can $\dagger$ be extended so that there results a continuous one to one correspondence $\pi$ between $G$ and $J+I$ such that for uncountably many points $P$ of $J$ it is true that $\pi(P)$ contains $P$.

Now only a countable number of elements of $G$ contain domains. Let $H$ denote $J+I$ and let $x$ denote any point of $H$. Let $C_{1}(x)$ denote $x$. Let $C_{2}(x)$ be the continuum $\pi(x)$. Let $C_{3}(x)$ denote the sum of all continua $\pi(y)$ for all points $y$ of $C_{2}(x)$. In general let $C_{n+1}(x)$ denote the sum of all elements $\pi(y)$ for every point $y$ of $C_{n}(x)$. If $x$ and $y$ are distinct points of $H$, then $C_{n}(x)$ and $C_{n}(y)$ are mutually exclusive continua. Hence, for each $n$, the set of all points $x$ such that $C_{n}(x)$ contains a domain is countable. Hence there exist two points $P_{1}$ and $P_{2}$ of $J$, and a simple continuous arc $T$ from $P_{1}$ to $P_{2}$, such that if $x$ is any point of $T$ then, for every $n, C_{n}(x)$ contains no domain, and $\pi\left(P_{i}\right)$ contains $P_{i}(i=1,2)$.

There exists an infinite set of simple closed curves $J_{1}, J_{2}$, $J_{3}, \cdots$ such that (1) $J_{1}=J$, (2) $J_{1} \cdot J_{2}$ is the point $P_{2}$, and in general, for each $i, J_{i} \cdot J_{i+1}$ is a single point $P_{i+1}$, and $J_{i} \cdot J_{i+k}$ $=0(k>1)$, (3) no point of $J_{k}$ lies within $J_{i}$, and (4) only a finite number of the curves $J_{1}, J_{2}, J_{3}, \cdots$ have points within any circle. For each $i$ let $\pi_{i}$ be a continuous transformation throwing

$t_{1}, t_{2}, t_{3}, \cdots$ satisfying (1) and (2) above, and such that $J-\sum_{i=1}^{\infty} t_{i}$ is uncountable, and indeed if $v(J)-\sum_{i}^{\infty} v\left(s_{i}\right)$ is a positive number $e$, then the segments $t_{1}, t_{2}, t_{3}, \cdots$ can be so chosen that the measure of the set $J-\sum_{i=1}^{\infty} t_{i}$ is as near $e$ as we please. If $P$ is any point of $J-\sum_{i=1}^{\infty} t_{i}$, then let $C(P)$ denote $P$. Consider every interval $s_{j}$ that is a subset of $t_{i}$ as an element, and every other point of $\tilde{t}_{i}$ as an element. Then the collection of elements so obtained is an arc, and can be made to correspond to the arc $\bar{t}_{i}$ of $J$. Thus if $T$ denotes the collection of intervals $s_{1}, s_{2}, s_{3}, \cdots$ and all other points of $J$, then there exists a correspondence $C$ such that (1) $C(T)=J$ and (2) for uncountably many points $P$ of $J$ the point $P$ is an element of $T$, and $C(P)=P$. But if $x$ is any element of $T$, then there is a continuum $g_{x}$ of $G^{*}$ containing $x$, and the correspondence $D$ throwing $g_{x}$ into $x$, for every element $x$ of $T$, is continuous. Then if $g$ is an element of $G^{*}$ the correspondence throwing $g$ into $C[D(g)]$ is a continuous one to one correspondence between $G^{*}$ and $J$ and satisfies the required conditions.

$\dagger$ See Schoenflies, Beiträge zur Theorie der Punktmengen, Mathematische Annalen, vol. 62 (1906), pp. 286-328. See also J. R. Kline, $A$ new proof of $a$ theorem due to Schoenflies, Proceedings of the National Academy of Sciences, vol. 6 (1920), pp. 529-531. 
$H$ into $J_{i}$ plus its interior in such a way that $\pi_{i}\left(P_{1}\right)=P_{i}$, and $\pi_{i}\left(P_{2}\right)=P_{i+1}$. For each $n$ let $E_{n}$ denote the sum of all continua $C_{n}(x)$ for every point $x$ of the $\operatorname{arc} T$. Note that $E_{n+1}$ can be obtained by adding together all continua $\pi(y)$ for all points $y$ of $E_{n}$. Let $M_{n}$ denote $\pi_{n}\left(E_{n}\right)$ and let $M$ denote $M_{1}+M_{2}+M_{3}+\cdots$. Then $M$ is the continuum desired.

Since no one of the sets $M_{1}, M_{2}, M_{3}, \cdots$ contains a domain, the continuum $M$ contains no domain. Let $R$ denote $P_{1}+P_{2}$ $+P_{3}+\cdots$ and let $x$ denote any point of $M-R$ belonging to $M_{i}(i>1)$. Let $y_{x}$ be the point of $H$ such that $\pi_{i}\left(y_{x}\right)=x$. Let $g_{y_{x}}$ denote the element of $G$ corresponding, under $\pi$, to the point $y_{x}$, and let $h_{x}$ denote the continuum $\pi_{i}\left(g_{y_{x}}\right)$. In case $h_{x}$ does not contain $P_{i}$ or $P_{i+1}$, then let $k_{x}$ denote $h_{x}$. For each $i(i>2)$ there is a point $x_{i-1}$ of $M_{i-1}$, and a point $\bar{x}_{i}$ of $M_{i}$ such that both the sets $h_{x_{i-1}}$ and $h_{x_{i}}$ contain $P_{i}$. Let $k_{P_{i}}$ denote $h_{x_{i-1}}+h_{\bar{x}_{i}}$. For some point $x$ of $M_{2}$ the set $h_{x}$ contains $P_{2}$. Let $k_{P_{2}}$ denote $h_{x}$ plus the $\operatorname{arc} T$. Then $M$ is the sum of the elements of an upper semicontinuous collection $G^{\prime}$ of mutually exclusive continua, every element of $G^{\prime}$ being a continuum $k_{Q}$ for some point $Q$ of $M$. The elements $k_{P_{3}}, k_{P_{4}}, k_{P_{6}}, \cdots$ are each homeomorphic with the sum of two elements of $G$ and every other element of $G^{\prime}$ except $k_{P_{2}}$ is homeomorphic with some one element of $G$. Every element of $G^{\prime}$ is a nondegenerate continuum. For each $i(i>1)$ let $G_{i}{ }^{\prime}$ denote the collection of all elements $k_{P}$ of $G^{\prime}$ for all points $P$ of $M_{i}$. Then $G_{2}^{\prime}$ is homeomorphic with the arc $M_{1}, G_{3}{ }^{\prime}$ is homeomorphic with $M_{2}, G_{4}^{\prime}$ with $M_{3}$, and so on indefinitely. Moreover, $G_{i}{ }^{\prime}$ and $G_{i+1}^{\prime}$ have exactly one element in common, which corresponds to the common point of $M_{i-1}$ and $M_{i}(i>1)$. Thus $G$ and $M$ are homeomorphic.

A bounded continuum with the same property may be obtained if condition (4) satisfied by the curves $J_{1}, J_{2}, J_{3}, \ldots$ is replaced by the following: (4) $J_{1}, J_{2}, J_{3}, \cdots$ is a contracting sequence having $P_{1}$ as sequential limit point. A continuum so obtained will have exactly two complementary domains in the plane $S$.

The University of TeXas 